\title{
Die Behandlung der Retinopathia diabetica mit Lichtkoagulation
}

\author{
A. Wessing und G. MeYer-Sohwickerath
}

Augenklinik am Klinikum Essen der Ruhruniversität Bochum (Direktor Prof. Dr. G. MEYER-SCHWIOKERATH)

Eingegangen am 7. Januar 1969

Treatment of diabetic retinopathy by light-coagulation Summary. Results of treating diabetic retinopathy by light-coagulation are reported. Early symptoms, e.g. microaneuryms, lipid deposits and retinal oedema, improved after light-coagulation. Coagulation during early phases of the disease prevented development of vascular proliferation, haemorrhages and retinal detachment. Where vascular proliferation and fibrous changes of the vitreous body were already present when the therapy was instituted, the further deleterious course was prevented in only a few cases.

Traitement de la rétinopathie diabétique par la photocoagulation

Résumé. Ce travail concerne le traitement de la rétinopathie diabétique par la photocoagulation. Les symptômes précoces, tels les microanévrismes, les dépôts lipidiques et les oedèmes de la rétine s'améliorent après photocoagulation. En utilisant la coagulation au stade précoce, on peut prévenir l'évolution vers la prolifération des vais. seaux, les hémorragies et le décollement de la rétine. Si au début du traitement il y a déjà des proliférations des vaisseaux et des altérations fibreuses du corps vitré, il est généralement trop tard pour pratiquer la photocoagulation, et un développement délétère ne pout être empêché que dans peu de cas.

Zusammenfassung. Es wird über die Behandlung der diabetischen Retinopathie mit Lichtkoagulation berichtet. Frühe Symptome, wie Mikroaneurysmen, Lipoidablagerungen und Netzhautödem bilden sich nach Lichtkoagulation zurück. Bei Koagulation im Frühstadium kann das Fortschreiten zu Gefäßproliferationen, Blutungen und Netzhautablösung verhindert werden. Sind bei Behandlungsbeginn bereits Gefäßproliferationen und fibröse Veränderungen des Glaskörpers vorhanden, kommt die Lichtkoagulation im allgemeinen zu spät, und ein weiterer deletärer Verlauf kann nur in wenigen. Fällen verhindert werden.

Key-words: Retinopathia diabetica, light-coagulation.

\section{Einführung}

Zum ersten Male wurden in den Jahren 1955-1959 diabetische Retinopathien mit Lichtkoagulation behandelt. Meyer-Schwickeratir hat 1959 in seiner Monographie über die Tichtkoagulation darüber berichtet. Es handelte sich um insgesamt 5 Fälle. Vier davon im proliferativen Stadium konnten nicht beeinflußt werden, einer dagegen mit ausschließlich Mikroaneurysmen und gelben Exsudaten zeigte eine deutliche Besserung.

In den folgenden Jahren wurden die Versuche, die diabetische Retinopathie mit Lichtkoagulation anzugehen, fortgesetzt. Das ursprüngliche Ziel war, rezidivierende Blutungen aus Mikroaneurysmen und neugebildeten Gefäßen zu unterbinden und durch disseminierte Koagulationen einer Netzhautablösung vorzubeugen. Die Versuche waren zunächst zögernd und tastend. Trotz einiger eindrucksvoller Ergebnisse schien zunächst Skepsis am Platz. Das Problem, Therapieergebnisse bei einer Krankheit zu beurteilen, die von einem Fall zum anderen so sehr unterschiedlich verläuft und bei der Spontanremission vorkommen, bedarf keiner Erläuterung.

Grundsätzlich bestätigten sich die durchweg positiven Ergebnisse jedoch immer wieder. Zur gleichen Zeit wurde auch von anderen Autoren die Lichtkoagulation zur Behandlung der diabetischen Retinopathie empfohlen. (Movra-BrazIL u. DT Rezende, 1964; Okun u. Clbis, 1966; Okun, 1968; Whtzig u. Jepson,
1966, 1967, 1968; Amalric u. Biad, 1967; GuInladMAT, 1968). 1963/64 hat K. Sснотт zum ersten Mal das gesamte Material der Essener Augenklinik zusammen. gestellt und es 1964 vor der Deutschen Ophthalmologischen Gesellschaft in Heidelberg präsentiert. Zu dieser Zeit zeichnete sich bereits die gute Wirksamkeit der Lichtkoagulation bei den frühen Stadien der Erkrankung, die noch keine Gefäßproliferationen aufweisen, ab. Es wurden daraufhin in den folgenden Jahren mehr und mehr diabetische Retinopathien mit Lichtkoagulation behandelt, und das Augenmerk richtete sich besonders auf die Erfassung von Frühfällen.

\section{Methode}

Die Lichtkoagulationen werden mit dem XenonKoagulator durchgeführt. Koaguliert werden Mikroaneurysmen, Gefäßneubildungen im Netzhautniveau und eventuell auch kleinere, eben beginnende Proliferationen in den Glaskörper. Die Koagulationen werden als Einzelherde gesetzt. Zwischen den einzelnen Koagulationen bleibt jeweils eine Netzhautbrücke unangetastet. Größere und besonders fächerförmige Gesichtsfelddefekte können so vermieden werden. Der Durchmesser einer Koagulation beträgt wahlweise zwischen 1.5 und $3^{\circ}$. Die Intensität der Koagulation wird so gewählt, daß es eben zu einer deutlichen Weilfärbung der Netzhaut kommt. Von der Macula wird ein Abstand von etwa $1 \frac{1}{2}$ bis 2 PD eingehalten. Koagulationen im papillomaculären Bündel bedeuten nicht unbe- 
dingt eine Gefährdung des zentralen Visus. Kleine Feldblende und geringe Koagulationsintensität sichern die Schonung der Nervenfasern.

Bei nicht zu schwerwiegendem Befall wird der ganze Augenhintergrund in einer Sitzung koaguliert. Dabei können bis zu 200 oder 300 Koagulationen vorgenommen werden. Sind die Veränderungen relativ weit fortgeschritten und sehr ausgedehnt, so wird die Behandlung auf 2 oder 3 Sitzungen verteilt. Die Koagulationen erfolgen im allgemeinen ambulant. Nur sehr schwere Fälle werden stationär aufgenommen.

Komplikationen sind bei nicht proliferativen Formen außerordentlich selten. Hämorrhagien kommen nur ganz vereinzelt vor. Erst mit dem Erscheinen von neugebildeten Gefäßen, die das Netzhautniveau verlassen haben und in den Glaskörperraum einsprossen, steigt die Gefahr postkoagulativer Blutungen rasch an. Eine zweite, weniger bedrohliche Komplikation ist das Auftreten einer exsudativen Netzhautablösung nach sehr ausgedehnter Koagulation. Diese pflegt sich im allgemeinen innerhalb weniger Tage zurückzubilden und ohne Folgen abzuheilen.

\section{Ergebnisse}

Bis Ende des Jahres 1967 verfügen wir über 127 Fälle mit diabetischer Retinopathie, die mit Lichtkoagulation behandelt wurden. 44 davon waren männliche Personen, 83 weibliche. 24 Patienten waren jünger und 103 älter als 40 Jahre. Insgesamt wurden 189 Augen behandelt. Im folgenden sind sie in Anlehnung an die 0'Hare-Klassifikation ${ }^{\mathbf{1}}$ aufgegliedert und in 2 Hauptgruppen untersucht und dargestellt.

\section{Gruppe $B$ (Back-ground-Retinopathie)}

Es finden sich Kapillarerweiterungen, Mikroaneurysmen, Gefäßneubildungen im Netzhautniveau, Netzhautödem, Lipoidablagerungen und intra- sowie präretinale, umschriebene Hämorrhagien.

\section{Gruppe $N$ (Neovaskularisation)}

Das Bild ist durch die Neubildung von Gefäßen und deren Einwachsen in den Glaskörperraum gekennzeichnet. Verbunden damit sind fibröse Verschwartung des Glaskörpers, Traktionsablatio und schwerste Blutungen.

Die Beobachtungszeit schwankt zwischen 9 Monaten und 7 Jahren. Der Durchschnitt der Fälle befindet sich seit 2,6 Jahren in regelmäßiger Kontrolle.

In Tabelle 1 sind die Behandlungsergebnisse nach dem ophthalmoskopischen Befund wiedergegeben. Es zeigt sich, daß die Ergebnisse für die Gruppe B, d.h. also für alle jene Fälle mit reiner „Back-ground-Retinopathie" sehr günstig sind. Von 129 Augen zeigen 81 eine entscheidende Besserung. Die Mikroaneurys-

1 Treffen eines Diabetologenausschusses auf dem Flughafen O'Hare in Chicago am 29. 6. 1968 auf Initiative des U.S. State Department of Health, Education and Welfare. men haben sich weitgehend zurückgebildet, die gelben Exsudate sind resorbiert und das Netzhautödem ist verschwunden. Bei 39 Augen ist der Prozeß stationär geblieben. Eine Progredienz dagegen mit Auftreten von Proliferationen und Blutungen findet sich lediglich bei 9 Fällen.

Ganz anders ist das Bild in der Gruppe der proliferativen Retinopathien N. Hier sind die Ergebnisse wesentlich ungünstiger. Lediglich 12 von 60 Augen zeigen eine eindeutige Besserung. Bei 34 Augen bleibt der Proliferationsvorgang unbeeinflu.Bt und 14 Augen wurden blind.

Betrachtet man nur die Fälle mit einer Beobachtungszeit von mehr als 3 Jahren (Tabelle 2), so ergibt sich ein ganz analoges Bild. Die Diskrepanz zwischen den reinen Back-ground-Formen und den proliferativen Retinopathien ist offensichtlich.

Tabelle 1. Ergebnisse der Lichthoagulation bei diabetischer Retinopathie. Beobachtungszeit zwischen 9 Monaten und 7 Jahren

$\mathrm{B}=$ Back-ground-Retinopathie

$\mathbf{N}=$ Retinopathie mit Gefäßneubildungen und Bindegewebsproliferationen.

\begin{tabular}{lllc}
\hline Klassifikation Bessorung & Stillstand & Progredienz \\
\hline $\mathrm{B}$ & 81 & 39 & 9 \\
\hline $\mathrm{N}$ & 12 & 34 & 14 \\
\hline
\end{tabular}

Tabelle 2. Ergebnisse der Lichtkoagulation bei diabetischer Retinopathie. Beobachtungszeit mehr als 3 Jahre

$\mathrm{B}=$ Back-ground-Retinopathie

$\mathrm{N}=$ Retinopathie mit Gefäßneubildungen und Bindegewebsproliferationen.

\begin{tabular}{llll}
\hline Klassifikation & Besserung & Stillstand & Progredienz \\
\hline $\mathrm{B}$ & 25 & 9 & 4 \\
\hline $\mathrm{N}$ & 1 & 7 & 2 \\
\hline
\end{tabular}

Dementsprechend ist auch das Verhalten des Visus bei nichtproliferativen und proliferativen Formen gegensätzlich. Bei den Fällen der B-Gruppe (Abb. 1) bleibt im großen und ganzen der vor der Behandlung vorhandene Visus erhalten. Einzelnen Fällen mit stärkerer Visusverschlechterung stehen andere mit deutlicher Visusbesserung gegenüber. Zum Unterschied dazu die Gruppe $\mathrm{N}$ (Abb. 2). Hier macht sich ein deutlicher Trend zur Sehverschlechterung bemerkbar. In einer ganzen Reihe von Fällen kommt es zu weitgehender Sehverschlechterung oder vollständiger Erblindung. Diese Entwicklung gilt sowohl für die Retinopathie des jugendlichen wie die des fortgeschrittenen Alters.

Komplikationen sind in der B-Gruppe selten, häufen sich dagegen in der Gruppe N. Bei 129 Fällen mit nichtproliferativer Retinopathie kommt es postkoagulativ lediglich $16 \mathrm{mal}$ zu Glaskörperblutungen. In der Gruppe $N$ dagegen kommen auf 60 Fälle 22 Blutungen, 
4 mal ein Sekundärglaukom, 3mal eine Ablatio exsudativa fugax und in 2 Fällen eine Opticusatrophie.

Es ist bemerkenswert, daß die Patienten postkoagulative Gesichtsfelddefekte subjektiv fast nie wahrnehmen. Die Ausfälle werden auch dann nur wenig bemerkt, wenn mehrere 100 Koagulationen durchgeführt wurden.

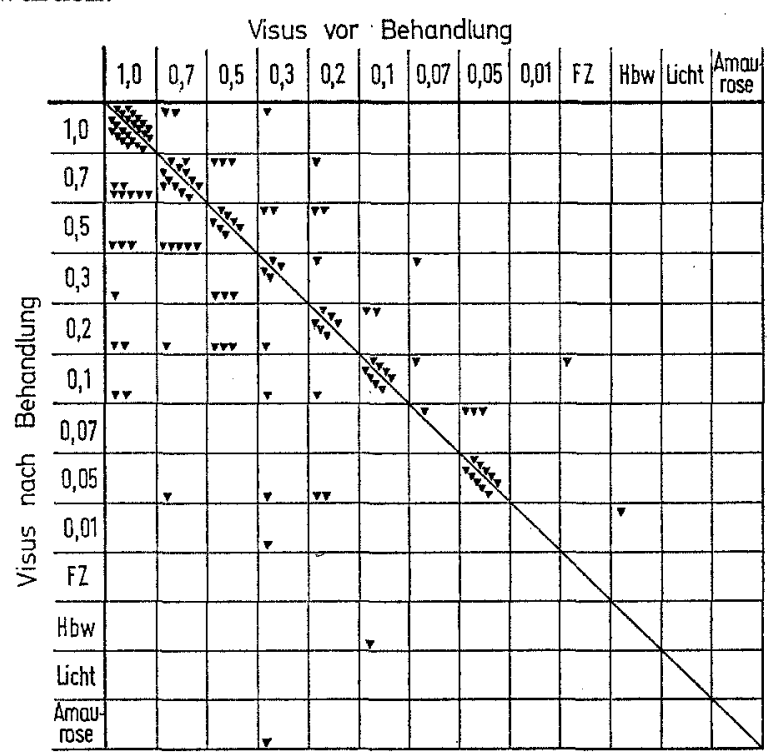

Abb. 1. Gruppe B (Back-ground-Retinopathie) Visus vor und nach Koagulation.

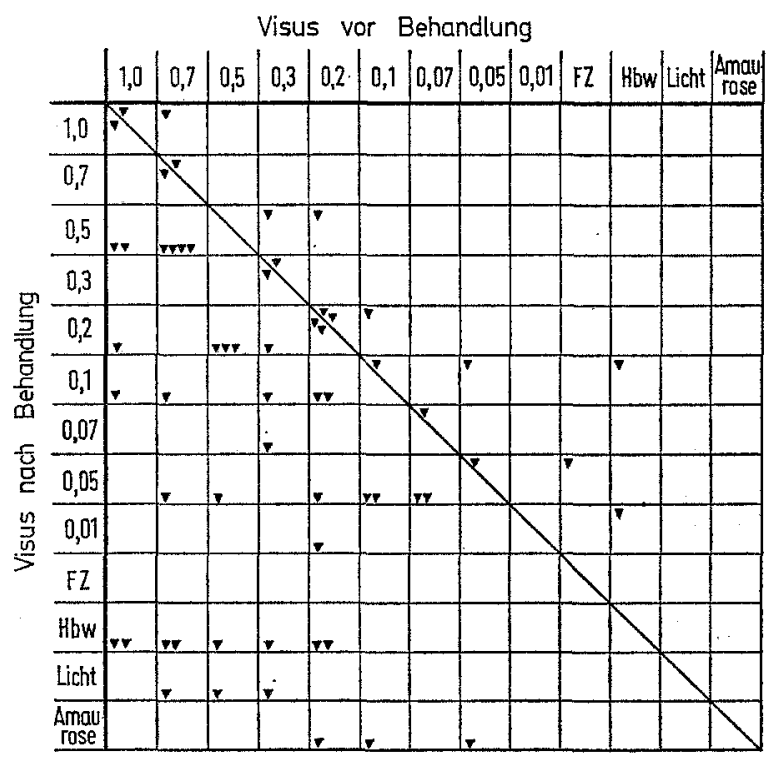

Abb. 2. Gruppe N (Retinopathie mit Gefäßproliferation) Visus vor und nach Koagulation

Die ovjektive Aufnahme der Gesichtsfelder bestätigt das. Sie ergibt meist nur eine geringfügige unregelmäßige Restriktion der Gesichtsfeldgrenzen. Auch bei den Fällen, wo kleine und umschriebene Koagulationen in der Nähe der Papille und um die Macula herum vorgenommen wurden, kommt es zu keinen wesentlichen Ausfällen.
Faßt man zusammen, so ergibt sich das folgende Bild: Die frühen Symptome der diabetischen Retinopathie sind reversibel, wenn die Lichtkoagulation vor dem Stadium der Gefäßproliferation durchgeführt wird. Durch Behandlung im Frühstadium lassen sich späte Folgen mit Proliferation, Blutung und Netzhautablösung verhindern. Bestehen zur Zeit des Behandlungsbeginns bereits Gefäßproliferationen und fibröse Veränderungen, so kommt die Lichtkoagulation in den meisten Fällen zu spät, und es gelingt nicht, das Fort. schreiten des Prozesses zu verhindern.

Bentham, 1963; Catrd u. Garret, 1963 sowie Fischer, 1966 haben über den natürlichen Verlauf der diabetischen Retinopathie berichtet. Die Prognose der mit Lichtkoagulation behandelten Fälle dürfte danach unzweifelhaft günstiger zu veranschlagen sein als ohne Therapie.

\section{Photographische Verlaufskontrolle}

Die Schwierigkeiten, die diabetische Retinopathie zu klassifizieren und aus einer solchen Klassifikation bindende Schlüsse auf die Behandlungsergebnisse zu ziehen, ist bekannt. Die statistische Analyse wird noch komplexer dadurch, daß das Material außerordentlich heterogen ist, weil sich die Indikationsstellungen immer wieder gewandelt haben. In dieser Situation kommt der vergleichenden Photographie eine besondere Bedeutung zu. Das Photo zeigt Schritt für Schritt, was mit den minutiösen Veränderungen am Gefäßsystem der Netzhaut und mit der Netzhaut selbst geschieht. Die Ergebnisse lassen sich von Bild zu Bild auf das Eindrucksvollste ablesen.

Unser Material wurde Fall für Fall gesichtet und die Fundusphotos, die oft in vielen Jahren Abstand. angefertigt worden sind, verglichen und ausgewertet. Selbstverständlich setzt die Photokontrolle über viele Jahre das Vorhandensein guter brechender Medien und eines klaren Funduseinblicks voraus. Damit ist von vornherein eine gewisse Auswahl in Richtung auf leichtere Fälle und Frühstadien gegeben. Die Ergebnisse sind die folgenden:

1. Schwere ödematöse Veränderungen der Netzhaut, welche auf den Bildern vor der Therapie oft die Aderhautzeichnung vollständig verdecken, verschwinden allmählich Monate und Jahre nach der durchgeführten Lichtkoagulation.

2. Koagulierte Mikroaneurysmen und Kapillarerweiterungen im Niveau der Netzhaut obliterieren und verschwinden.

3. Es bilden sich auch solche neuentstandenen Gefäße zurück, die nicht direkt koaguliert worden sind.

4. Gelbe, harte Exsudate sind in den meisten Fällen eindeutig rückläufig.

5. Das Volumen und die Schlängelung der Venen verringern sich.

6. Die Rückbildung der Veränderungen erfolgt beim Jugendlichen wesentlich schneller - nämlich innerhalb 
einiger Monate - als bei Patienten jenseits des 50. Lebensjahres. Das Endstadium der Besserung wird hier nicht selten erst nach etlichen Jahren erreicht.

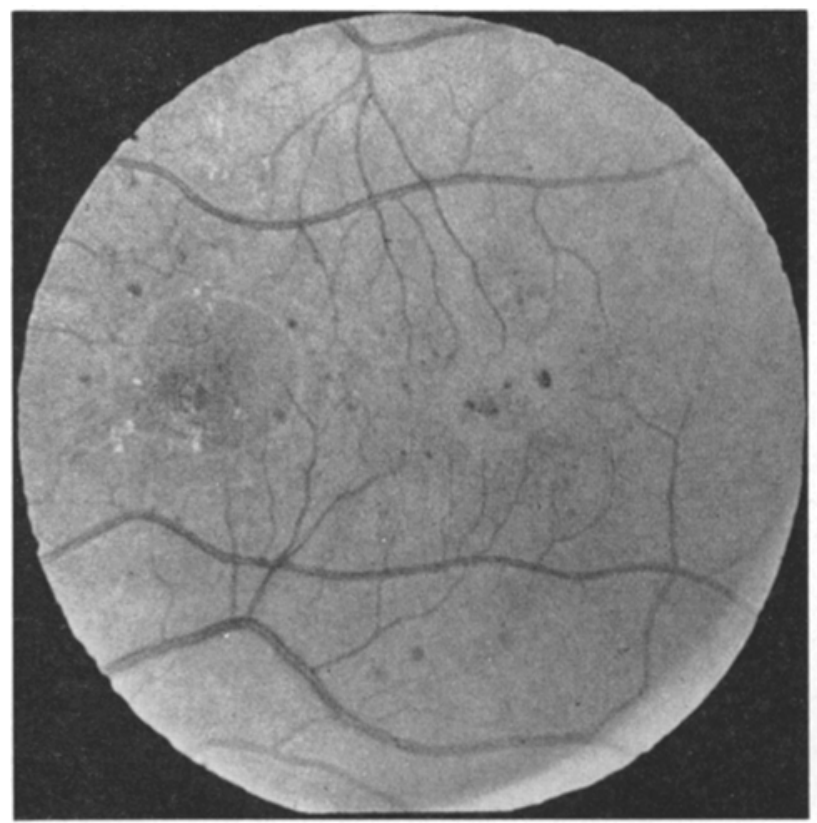

Abb. $3 a$

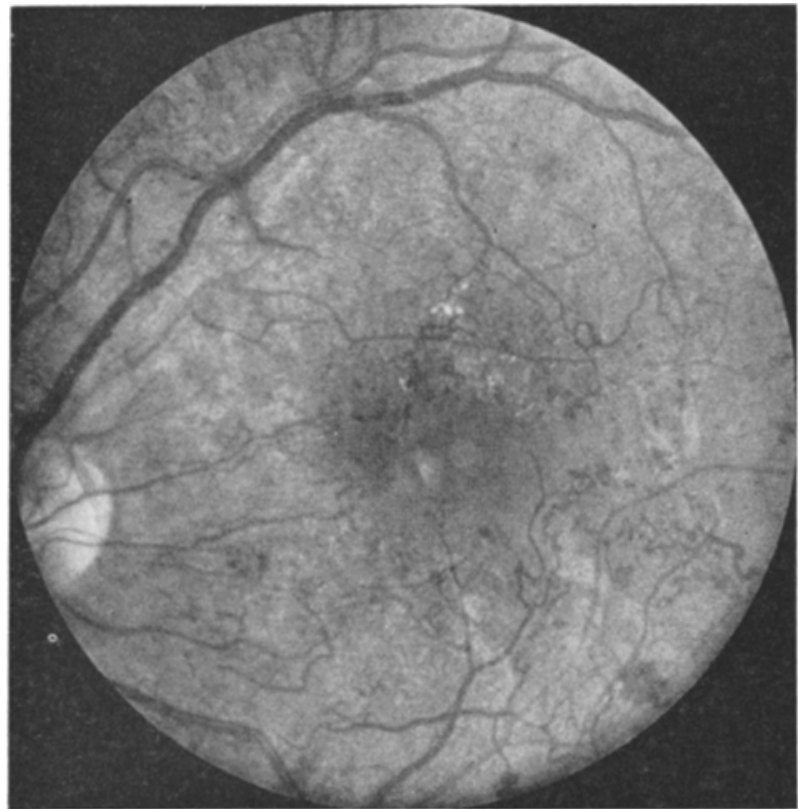

Abb. $4 a$

\section{Fall 1. Abb. 3a und b}

33-jährige Patientin, Diabetes seit dem 15. Lebensjahr bokannt.

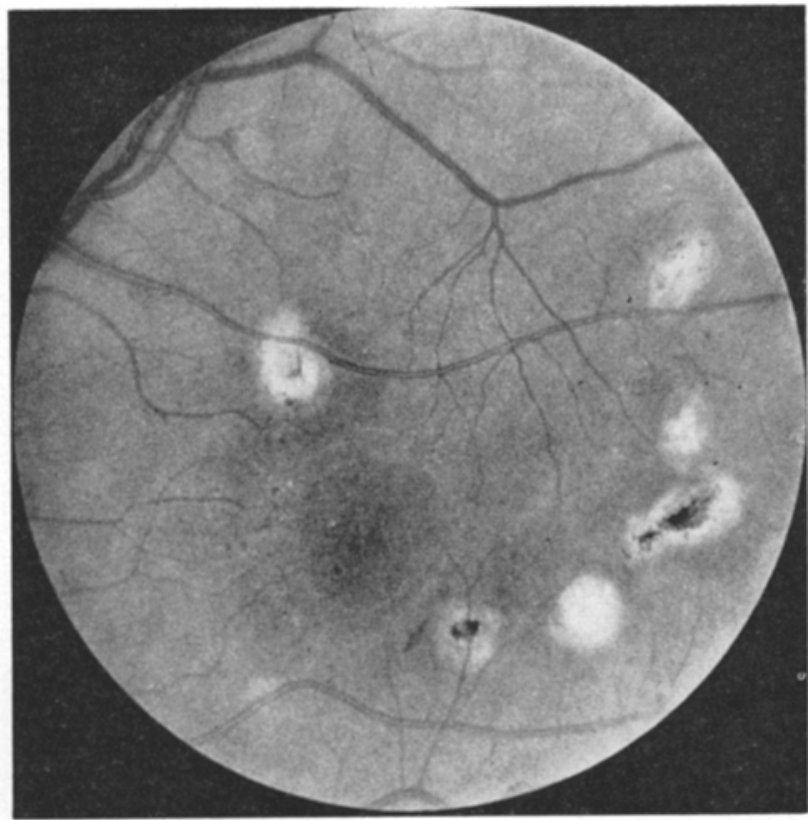

A.bb. $3 b$

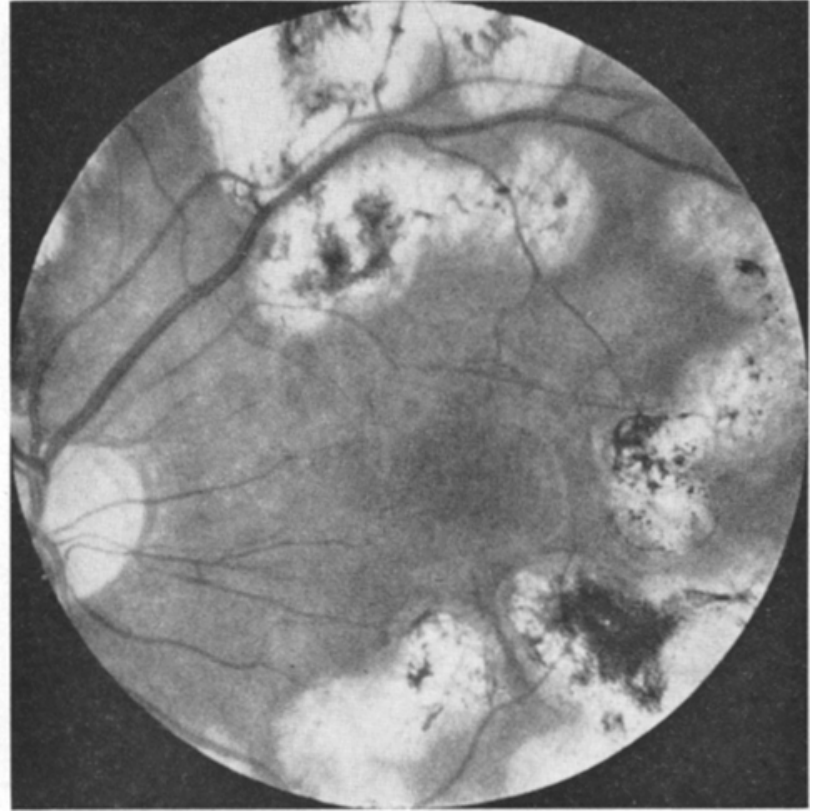

Abb. $4 \mathrm{~b}$

Abb. 3-4. s. Text, Fall 1 und 2

7. Die Lichtkoagulation verhindert die Entwicklung von Glaskörperproliferationen.

Im folgenden sollen anhand einiger Bilderserien diese Vorgänge dokumentiert werden.
Es findet sich eine diabetische Retinopathie mit ausschließlich Back-ground-Veränderungen, Unregelmäßigkeiten der Netzhautoberfläche, Mikroaneurysmen und kleineren Blutungen. Bild 3 a stammt aus dem Jahre 1963 und ist vor der Koagulation aufgenommen. Bild 3 b zeigt 
das Auge 3 Jahre später. Man sieht die Koagulationsnarben und erkennt, daß nur noch ganz vereinzelt Mikroaneurysmen und letzte Reste von gelben Exsudaten vor. handen sind.

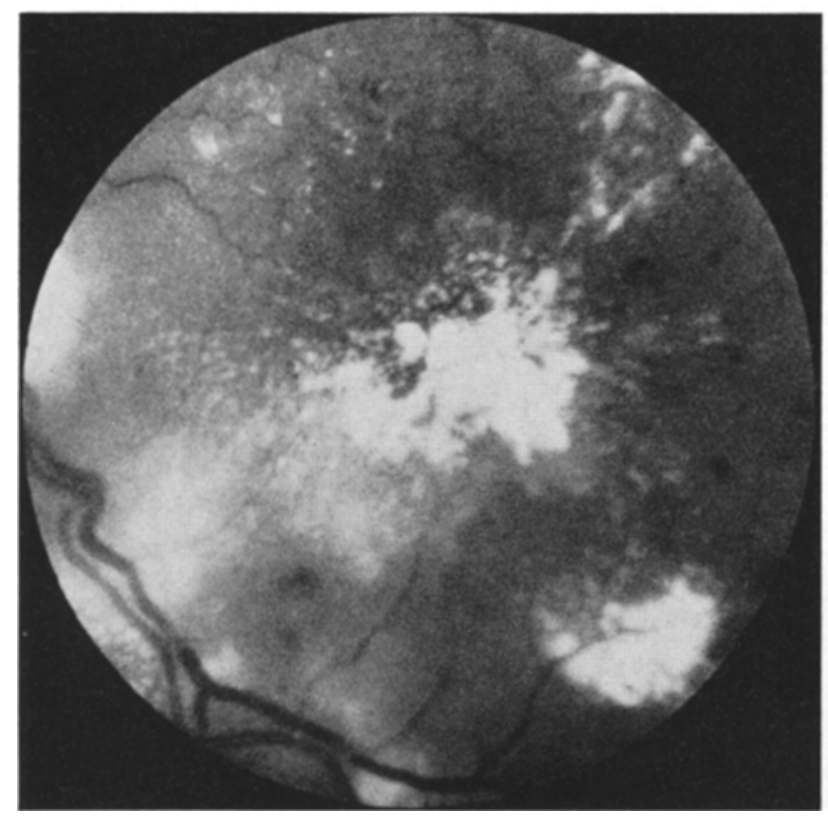

Abb. 5 a

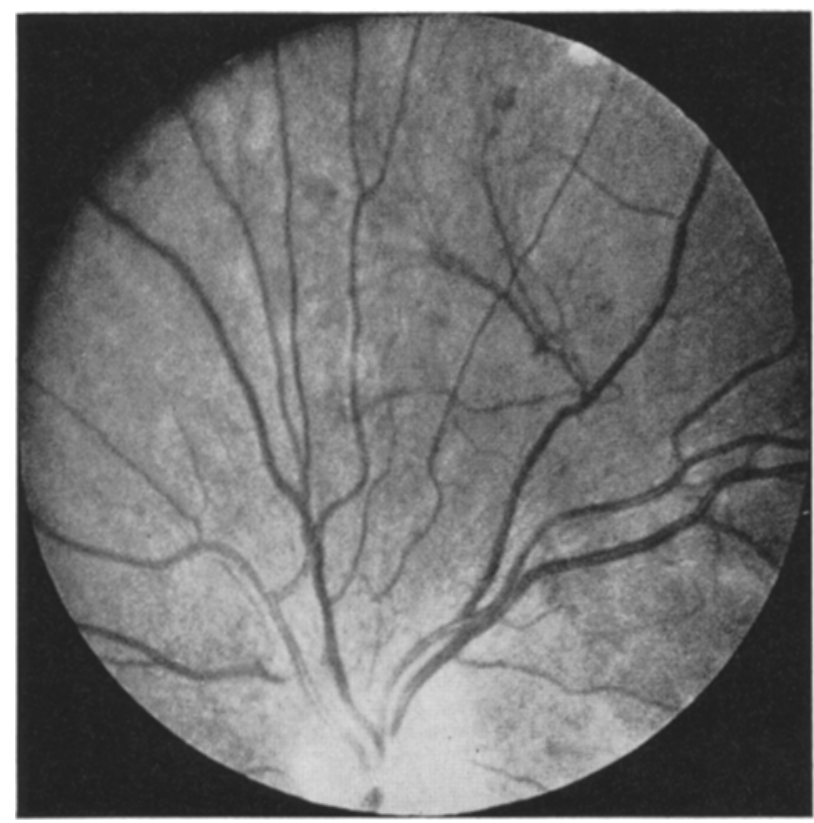

$\mathrm{Abb} .6 \mathrm{a}$ ten Kapillarerweiterungen, Gefäßnoubildungen im Netzhautniveau, zahlreichen Blutungen und Netzhautödem. Die Abb. 4a ist aus dem Jahre 1964 und zeigt den Befund unmittelbar vor der Koagulation. Das Bild $4 \mathrm{~b}$ ist 3 Jahre

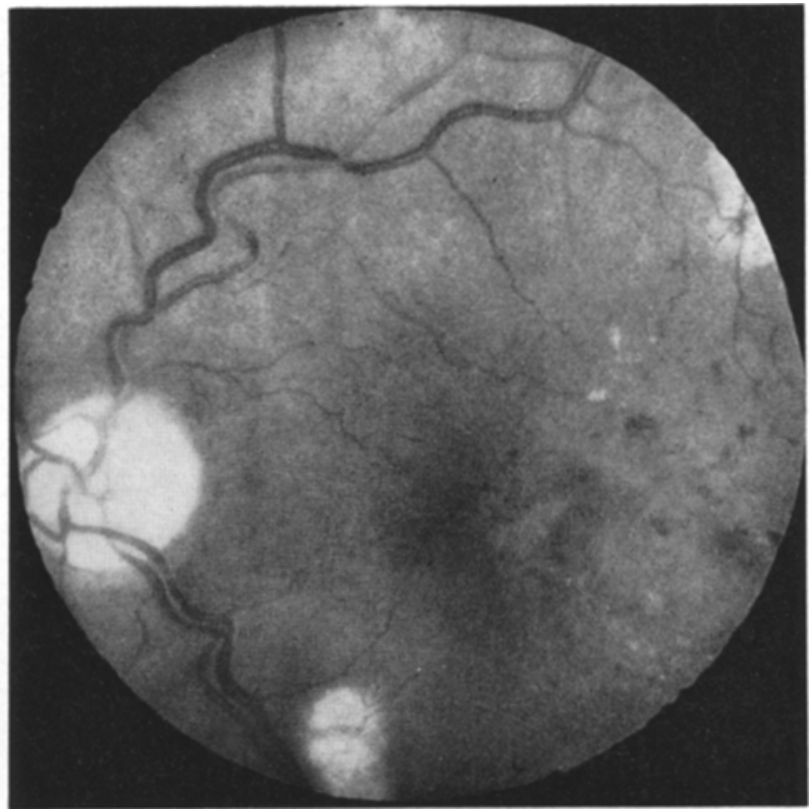

Abb. $5 b$

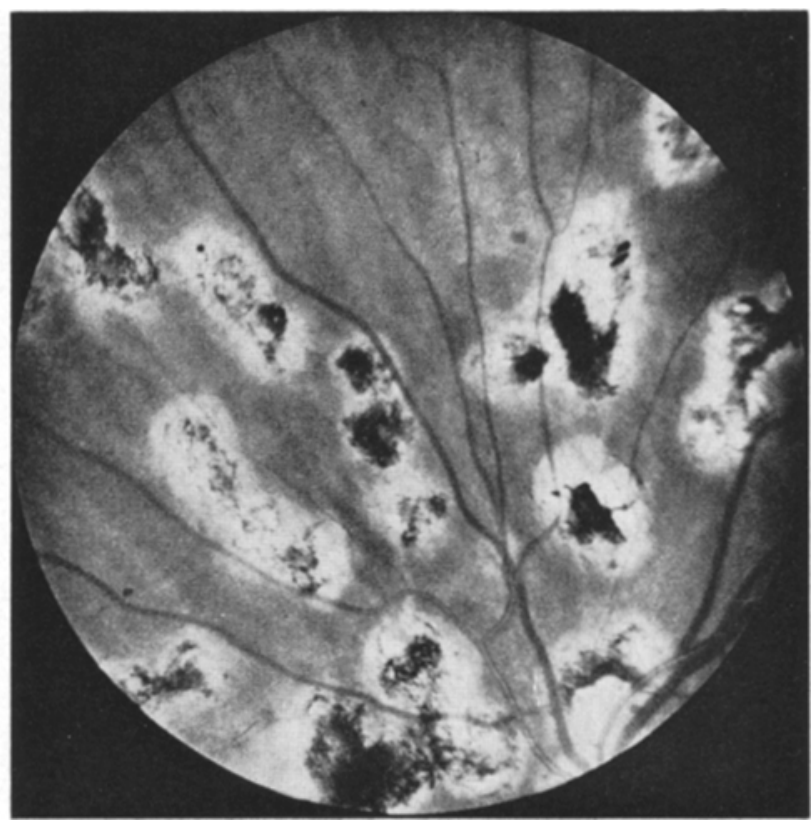

$\mathrm{Abb} .6 \mathrm{~b}$

Abb. $5-6$ s. Text, Fall 3 und 4

Fall 2. Abb. $4 \mathrm{a}$ und b

22-jährige Patientin, Diabetes seit 7 Jahren bekannt. Diese Serie gibt ein besonders eindrucksvolles Bild von der Wirksamkeit der Lichtkoagulation. Es findet sich eine schwere „Rubeosis retinae" (THIEL) mit flächenhaf- später aufgenommen worden. Das Netzhautödem ist völlig verschwunden. Die Aderhautzeichnung ist wioder zu sehen. Die neugebildeten Gefäße sind praktisch voll ständig verschwunden, die erweiterten Gefäße haben sich normalisiert. 
Fall 3. Abb. 5a und b

60 -jährige Patientin, Diabetes seit 10 Jahren bekannt. Diese Serie zeigt den Rückgang von gelben, harten Exsudaten. Das Bild 5 a aus dem Jahre 1960 zeigt vor der Koagulation eine massive Spritzerfigur im Maculabereich. Das zweite Bild (5b) 1963 läßBt erkennen, wie nach wenigen Koagulationen in weitem Abstand von der Macula dio Einlagerungen vollständig resorbiert worden sind.

\section{Fall 4. Abb. 6a und b}

24-jährige Patientin, Diabetes seit 10 Jahren bekannt. Diese Abbildungsserie demonstriert wie auch kleinere Gefäßproliferationen, die bereits die Netzhautebene verlassen haben, durch die Lichtkoagulation beseitigt werden können. Man erkennt in der Abb. 6a aus dem Jahre 1964 ein einzelnes Gefäßbäumchen, das sich zwischen den größeren Gefäßen ausspannt. Nach der Koagulation, wobei besonders darauf geachtet wurde, die Gefäße am Fuße des Fächers zu zerstören, verschwinden die Veränderungen vollständig. Bild $6 \mathrm{~b} 2$ Jahre später zeigt, daß die Zerstörung gelungen ist. Das Gefäßbäumchen ist vollständig verschwunden. $\mathrm{Zu}$ erneutem Gefäßwachstum ist es nicht gekommen.

\section{Diskussion und Schluß}

Der Wirkungsmechanismus der Lichtkoagulation auf die diabetische Angiopathie ist bisher unbekannt. Die hier dargelegten Beobachtungen werfen einige Schlaglichter auf diese Frage. Es ist zweifelhaft, ob die direkte Koagulation der Mikroaneurysmen und der geschädigten Netzhautkapillaren überhaupt Ursache für die Besserung der diabetischen Retinopathie ist. Wenn sich Gefäße, die nicht selbst koaguliert worden sind, normalisieren können, dann muß diesem Vorgang ein anderer Mechanismus zugrunde liegen. Mrurus, AmaLRIC, OKUN u.a. haben gezeigt, daß eine diabetische Retinopathie durch eine einseitige hohe Myopie, durch Opticusatrophie, disseminierte Chorioretinitis, durch ausgedehnte diathermische Narben nach Ablatiooperationen oder durch einseitige Erniedrigung des Ophthalmicadrucks verhindert werden kann. Es wäre denkbar, daß der Effekt der Lichtkoagulation bei der diabetischen Retinopathie Folge der Zerstörung normalen Netzhautgewebes ist, durch die wiederum die Stoffwechselaktivität und damit der Sauerstoffbedarf der gesamten Retina herabgesetzt werden. Amariric hat diese Ansicht bereits vor Jahren vertreten. Es sei in diesem Zusammenhang auf die fluoreszenzangiographischen Untersuchungen von HIckay $u$. Fraser hingewiesen, die eine deutliche Herabsetzung des Stromzeitvolumens in der Netzhaut bei diabetischer Retinopathie festgestellt haben.

Zweifellos ist die Behandlung der diabetischen Retinopathie mit Lichtkoagulation symptomatisch und nicht spezifisch. Doch solange keine wirksameren und für den Gesamtorganismus weniger gefährliche Maßnahmen zur Verfügung stehen, sollte man nach unserer Meinung nicht zögern, sie großzügig einzusetzen.

\section{Literatur}

Amalric, P.: Essai de traitement de la rétinopathie diabétique exsudative. Bull. Soc. Ophtal. Fr. 5, 359-363 (1960).
- Nouvelles considérations concernant l'évolution et le traitement de la rétinopathie diabétique. Ophthalmologica (Basel) 154, 151-160 (1967).

-, et C. BIAU: La diathermiecoagulation et la photocoagulation dans le traitement de la rétinopathie diabétique. Arch. Ophtal. (Paris) 27, 553-584 (1967).

Beetham, W.P.: Visual prognosis in proliferating diabetic retinopathy. Brit. J. Ophthal. 47, 611-619 (1963).

CAIRD, F.I., and I.C. Garret: Prognosis for vision in diabetic retinopathy. I. diabetes Radcliffe inform. Oxford 12, 389-397 (1963).

Frscher, R.: Die diabetische Retinopathie im Lebensflusse (Biorheutische Nosologie von Max Bürger). Ber. dtsch. ophthal. Ges. 60, $190-196$ (1956).

- Eine besondere Verlaufsform der Retinopathia diabetica. Albrecht v. Graefes Arch. Ophthal. 167, 607613 (1964).

Guillaumat, L.: Indications et limites de la photocoagulation dans le traitement de la rétinopathie diabétique. Bibl. ophthal. (Basel) 76, 154-159 (1968).

-, et A. Esta: Indications et limites de la photocoagulation dans le traitement de la rétinopathie diabétique. Ann. Oculist. (Paris) 201, 516-526 (1968).

Hickam, J.B., and R. Frayser: Studies of the retinal circulation in man. Observations on vessel diameter, arteriovenous oxygen difference and mean circulation time. Circulation 33, 302-316 (1966).

Lopez de Andrade, A. et S. De SÂ: Alguns resultados clinicos da fotocoagulascão. Arch. port. Oftal. 14, $63-73$ (1962).

Meyer-SChwickerath, G.: Lichtkoagulation. Bücherei des Augenarztes. 33. Beiheft zu Klin. Mbl. Augenheilk. Stuttgart: Enke, 1959.

-- Photocoagulation in diabetic retinipathy. Amer. J. Ophthal. 66, 597-603 (1968).

Moura-BraziL, N., et J. Rezende: Le rôle de la photocoagulation en ophtalmologie. Bull. Soc. frane. Ophtal. 74, 699-710 (1961).

OKUn, E.: The effectiveness of photocoagulation in the therapy of proliferative diabetic retinopathy. Amer. Acad. Ophthal. Otolaryng. 72, 246-252 (1968).

- , and P.A. CIBIS: The role of photocoagulation in the therapy of proliferative diabetic retinopathy. Arch. Ophthal. 75, 337-352 (1966).

Sснотт, K.: Weitere Erfahrungen mit der Lichtkoagulation bei Retinopathia diabetica. Ber. dtsch. ophthal. Ges. 66, 349-354 (1965).

Wetzig, P. C., and C. N. Jepson: Treatment of diabetic retinopathy by light-coagulation. Amer. J. Ophthal. 62, $459-465$ (1966).

- - Further observations on the treatment of diabetic retinopathy by light-coagulation. Amer. Acad. Ophthal. Otolaryng. 71, 902-906 (1967).

- The intravenous use of fluorescein as an adjunct in light-coagulation. Mod. Probl. Ophthal. (Basel) 7, 276278 (1968).

-, and J.P. WorLTom: Treatment of diabetic retinopathy by light-coagulation. Brit. J. Ophthal. 47, 539$541(1963)$.

Priv.-Doz. Dr. A. Wessing

Prof. G. Meyer-Schwickerath

Augenklinik am Klinikum Essen

der Ruhr-Universität Bochum

43 Essen-Holsterhausen

Städt. Krankenhaus

Hufelandstr. 55 